\title{
Low Level of Vitamin D is Correlated with High C-Reactive Protein (CRP) and Disease Activity in Indonesian Juvenile Idiopathic Arthritis (JIA) Patients
}

\author{
Desy Wulandari*, Wisnu Barlianto, Tita Luthfia Sari \\ Department of Pediatric Allergy Immunology, Saiful Anwar Hospital, Faculty of Medicine, Universitas Brawijaya, Jl. Veteran, Malang, Indonesia \\ *Corresponding author. E-mail: desywulandari@ub.ac.id
}

Received date: Jan 24, 2020; Revised date: Apr 14, 2020; Accepted date: Apr 23, 2020

\section{Abstract}

$\mathrm{B}$ ACKGROUND: Vitamin D plays essential role in the regulation of inflammation, such as in pathogenesis of Juvenile Idiopathic Arthritis (JIA). Vitamin D deficiency has been reported among JIA patients, but there were conflicting results regarding the correlation with disease activity. This study aimed to assess vitamin D serum level and its correlation with C-Reactive Protein (CRP) and disease activity in JIA patients.

METHODS: Children who were diagnosed with JIA according to International League of Associations for Rheumatology (ILAR) criterias were enrolled as JIA group subjects, while age and sex-matched healthy children were enrolled as the control group subjects. Vitamin D and CRP serum level were measured. Disease activity of JIA patients was calculated by Juvenile Arthritis Disease Activity Score-27 (JADAS-27).
RESULTS: Vitamin D serum level was lower in the JIA group compared to the healthy control group $(p=0.000)$. Among 26 JIA patients, $61.5 \%$ were deficient, $30.8 \%$ were insufficient, and $7.7 \%$ had normal vitamin D. No significant different in CRP level between vitamin D group $(p=0.441)$, but there was significant different in JADAS-27 $(p=0.001)$. The mean of CRP and JADAS-27 were found highest in vitamin D deficiency group. Vitamin D serum level was negatively correlate with CRP $(p=0.021, \mathrm{r}=-0.452)$ and JADAS-27 $(p=0.001 \mathrm{r}=-0.595)$.

CONCLUSION: Low level of vitamin D in JIA patients was inversely related to higher CRP and disease activity, suggesting that vitamin D supplementation could be have potential role in JIA treatment.

KEYWORDS: vitamin D, CRP, disease activity, JADAS-27, JIA

Indones Biomed J. 2020; 12(2): 149-56

\section{Introduction}

In recent decades, it has been established that vitamin $\mathrm{D}$ plays an important role in the regulation of inflammation, thus implicating vitamin D deficiency in the pathogenesis of several autoimmune and chronic rheumatic diseases. (1) Juvenile idiopathic arthritis (JIA) is an autoimmune, chronic inflammatory joint disease and is the most common type of arthritis in children.(2) It causes persistent joint pain, swelling, and stiffness and often leads to substantial longterm morbidity and physical disability.(3)

The International League of Associations for Rheumatology (ILAR) criteria define JIA as arthritis that arises before 16 years of age and persists for at least six weeks, with the exclusion of other known conditions.(4) Several studies have reported low levels of vitamin D in children with JIA (5-7), and it has been suggested that low vitamin D levels are correlated with higher disease activity (8). A cohort study conducted in children with JIA showed that a low level of vitamin D was associated with higher disease activity and increased risk of developing uveitis.(9) A similar study performed in Turkey reported that vitamin D was negatively correlated with disease activity in JIA patients.(10)

Several recent publications have highlighted the potential role of vitamin $\mathrm{D}$ in regulating inflammation. According to a study conducted in Kuwait, JIA patients with vitamin $\mathrm{D}$ deficiency and high $\mathrm{C}$-reactive protein (CRP) levels produced significantly higher levels of 
proinflammatory cytokines such as tumor necrosis factor (TNF)- $\alpha$ and interleukin (IL)- 8 than subjects with low CRP levels.(11) CRP is an acute-phase protein produced by the liver that increases in response to inflammation. Further, the ratios of anti-inflammatory/pro-inflammatory cytokines in patients with low levels of CRP suggest that vitamin D plays a role in maintaining an anti-inflammatory environment, as the ratios are higher in patients with nondeficient vitamin $\mathrm{D}$ levels than in patients deficient in vitamin $\mathrm{D}$. These data point to a possible role for vitamin $\mathrm{D}$ in the regulation of inflammation.(11) However, other studies found no correlation between vitamin D levels and JIA subtypes or disease activity.(12-15) A meta-analysis revealed that vitamin D supplementation had no effect on CRP and pro-inflammatory cytokines.(16) Given these conflicting results, it is essential to understand the role of vitamin D in JIA to optimize the management of JIA patients. This is the first study in Indonesia concerning vitamin D and disease activity in children with JIA. This study aimed to assess vitamin D serum levels and their correlation with CRP and disease activity in patients with JIA in Indonesia.

\section{Methods}

\section{Research Subjects}

This study was conducted between January 2017 and December 2019 at the Pediatric Allergy and Immunology Department at Saiful Anwar Hospital, Malang, Indonesia. Children aged 5-16 years who were diagnosed with JIA and had routine clinical monitoring at Saiful Anwar Hospital were enrolled. The ILAR classifies JIA patients into different subtypes, including oligoarthritis, rheumatoid factor-positive polyarthritis, rheumatoid factor-negative polyarthritis, and systemic-onset arthritis.(2) Subjects who had concurrent medical problems (malignancy, type 1 diabetes, inflammatory bowel disease, autoimmune disease, and immunodeficiency), had a history of recent infection (within two weeks), or were using any medications that would affect vitamin D metabolism were excluded from this study. The healthy control group consisted of ageand sex-matched participants who were undergoing a routine health examination. This research was approved by Ethical Committee of the Faculty of Medicine, Brawijaya University, Indonesia (236/EC/KEPK/10/2017).

\section{Blood Sampling}

Venous blood samples were collected into vacutainer tubes for complete blood count examination and serum analysis.
The coagulated blood was centrifuged for 10 minutes at 3,000 rpm; the serum was aliquoted into sterile tubes and stored frozen at $-20^{\circ} \mathrm{C}$ until analysis.

\section{Measurement of Vitamin D and CRP}

Serum vitamin D levels (25-hydroxy vitamin D; 25-(OH) D) were measured by enzyme-linked immunosorbent assay (ELISA) with a commercial 25-OH Vitamin D Human ELISA Kit (NovaTeinBio, Greater Boston, USA). The presence of vitamin D antigen in the sample will captured with an antigen-specific antibody. The detection antibody then covalently attached to enzypme linked secondary antibody. Enzyme substrate was then added to the wells producing a visible signal that is correlated with the amount of antigen and measured by a spectrophotometer. Vitamin D deficiency was defined as serum $25(\mathrm{OH}) \mathrm{D}<20 \mathrm{ng} / \mathrm{mL}$, insufficiency as 20-30 ng/mL, and sufficiency as $>30 \mathrm{ng} / \mathrm{mL}$.(17-19) The level of CRP in serum was measured using Cobas 6000 c501 Chemistry Analyzer immunoenzymometric assay (Roche Diagnostics, Holliston, USA). It used a single immunological reaction between CRP and peroxidase labeled antibody with another antibody attached to the wall of the test tube. The immune complex formed was determined by a colorimetric assay using a peroxidase substrate. The normal level of CRP in human serum was $2 \mathrm{mg} / \mathrm{L}$, whereas during inflammatory conditions, its concentration rises significantly.

\section{Measurement of Disease Activity}

Disease activity measurement in JIA patients was assessed using the Juvenile Arthritis Disease Activity Score (JADAS)-27. This score includes four measures: global physician assessment of disease activity using a visual analog scale (VAS), global parent assessment of child's well-being determined using a VAS, count of joints with active disease, and erythrocyte sedimentation rate (ESR). The physician assessment of disease activity was based on a $10-\mathrm{cm}$ VAS, where 0 corresponded to no activity and ten corresponded to maximum activity. The parent's assessment of the child's well-being was based on another 10-cm VAS in which 0 corresponded to very well and 10 to very poor. The count of active joints in JADAS-27 evaluated 27 joints: cervical spine, elbows, wrists, metacarpophalangeal joints (from first to third), proximal interphalangeal joints, hips, knees, and ankles. Each active joint scored 1 point, and the total ranges from 0 to 27 . ESR was normalized to a score ranging from 0 to 10 using the formula (ESR-20)/10. JADAS-27 was then calculated as the simple linear sum of the scores of its four components, which yields a total score of 0 to 57 , with higher scores representing worse 
disease activity.(20) The cutoffs for disease activity states based on JADAS-27 for oligoarthritis were inactive disease $\leq 1$, low disease activity $1.1-2$, moderate disease activity 2.1-4.2, high disease activity $>4.2$; while for polyarthritis and systemic-onset arthritis were inactive disease $\leq 1$, low disease activity $1.1-3.8$, moderate disease activity $3.9-8.5$, high disease activity $>8.5$.(21)

\section{Statistical Analysis}

Data analyses were performed using the software program SPSS Statistics, version 25.0 (IBM Corporation, New York City, USA). The $p$-values $\leq 0.05$ were considered significant. Descriptive statistics are reported as mean \pm standard deviation. Comparative analysis of parameters between the JIA and control groups was performed using the independent t-test and chi-square test for parametric data and the MannWhitney test for non-parametric data. Statistical analysis comparing parameters between the vitamin D groups was conducted using analysis of variance (ANOVA) for parametric data and the Mann-Whitney test for nonparametric data. The Pearson correlation test was used to analyze the associations between vitamin D, CRP, and JADAS-27. A multivariate linear regression analysis was performed to analyze the association between JADAS-27 and potential confounders.

\section{Results}

Fifty-one subjects including 20 boys and 31 girls, aged $12.29 \pm 2.02$ years old, were included in this study. There were 26 patients in the JIA group and 25 participants in the healthy control group. The demographic characteristics of the subjects are shown in Table 1. There was no difference in sex distribution or age between the groups. There was a higher incidence of underweight nutritional status in the JIA group compared to the control group. From the complete blood count analysis, the mean leukocyte and thrombocyte counts were higher in the JIA group.

There was a lower mean level of serum vitamin D in the JIA group compared to the healthy control group, $18.94 \pm 7.87 \mathrm{ng} / \mathrm{mL}$ and $27.38 \pm 6.39 \mathrm{ng} / \mathrm{mL}(p=0.000)$. Among JIA group, vitamin D deficiency insufficiency were found in $16(61.5 \%)$ and $8(30.8 \%)$ subjects, respectively. Only $2(7.7 \%)$ subjects were found to have adequate vitamin D levels. In the healthy control group, only $2(8 \%)$ subjects had vitamin D deficiency, 10 (40\%) subjects had vitamin D insufficiency, and 13 (52\%) subjects had sufficient levels of vitamin D.

Regarding JIA subtypes, there were 12 (46.1\%) subjects with oligoarthritis, $8(30.75 \%)$ subjects with

Table 1. Characteristics of study participants.

\begin{tabular}{|c|c|c|c|}
\hline Parameters & $\begin{array}{l}\text { JIA Group } \\
(\mathbf{n}=26)\end{array}$ & $\begin{array}{l}\text { Healthy Control } \\
\text { Group }(n=25)\end{array}$ & $p$-value \\
\hline \multicolumn{4}{|l|}{${ }^{\#}$ Sex, n } \\
\hline Female & 15 & 16 & \multirow{2}{*}{0.645} \\
\hline Male & 11 & 9 & \\
\hline s Age (years old) & $12.92 \pm 1.62$ & $10.65 \pm 2.08$ & 0.052 \\
\hline \multicolumn{4}{|l|}{${ }^{\prime}$ Nutritional status, $\mathrm{n}$} \\
\hline Well nourish & 9 & 20 & \multirow{2}{*}{$0.001 *$} \\
\hline Underweight & 17 & 5 & \\
\hline \multicolumn{4}{|l|}{ Laboratory results } \\
\hline${ }^{\$}$ Hemoglobin levels (g/dL) & $11.79 \pm 1.62$ & $12.45 \pm 0.69$ & 0.064 \\
\hline${ }^{\mathrm{S}}$ White blood cell count $\left(\mathrm{mm}^{3}\right)$ & $9537.69 \pm 2674.58$ & $7965.2 \pm 1271.32$ & $0.011^{*}$ \\
\hline${ }^{\$}$ Thrombocyte count $\times 10^{3}\left(/ \mathrm{mm}^{3}\right)$ & $401.73 \pm 107.54$ & $282.04 \pm 68.54$ & $0.000^{*}$ \\
\hline${ }^{\$}$ Vitamin D serum level $(\mathrm{ng} / \mathrm{mL})$ & $18.94 \pm 7.87$ & $27.38 \pm 6.39$ & $0.000^{*}$ \\
\hline${ }^{\$} \mathrm{CRP}$ serum level $(\mathrm{mg} / \mathrm{L})$ & $3.34 \pm 3.65$ & $0.72 \pm 0.57$ & $0.000^{*}$ \\
\hline \multicolumn{4}{|l|}{ Classification of vitamin $\mathrm{D}, \mathrm{n}$} \\
\hline Deficiency $(<20 \mathrm{ng} / \mathrm{mL})$ & 16 & 2 & \\
\hline Insufficiency (20-30 ng/mL) & 8 & 10 & \\
\hline Sufficiency $(>30 \mathrm{ng} / \mathrm{mL})$ & 2 & 13 & \\
\hline
\end{tabular}

Results are expressed as frequency and mean \pm SD. ${ }^{~}$ Tested with Chi-Square Test; ${ }^{\$}$ Tested with Independent T-test; ${ }^{*} p<0.05$ was considered significant. 


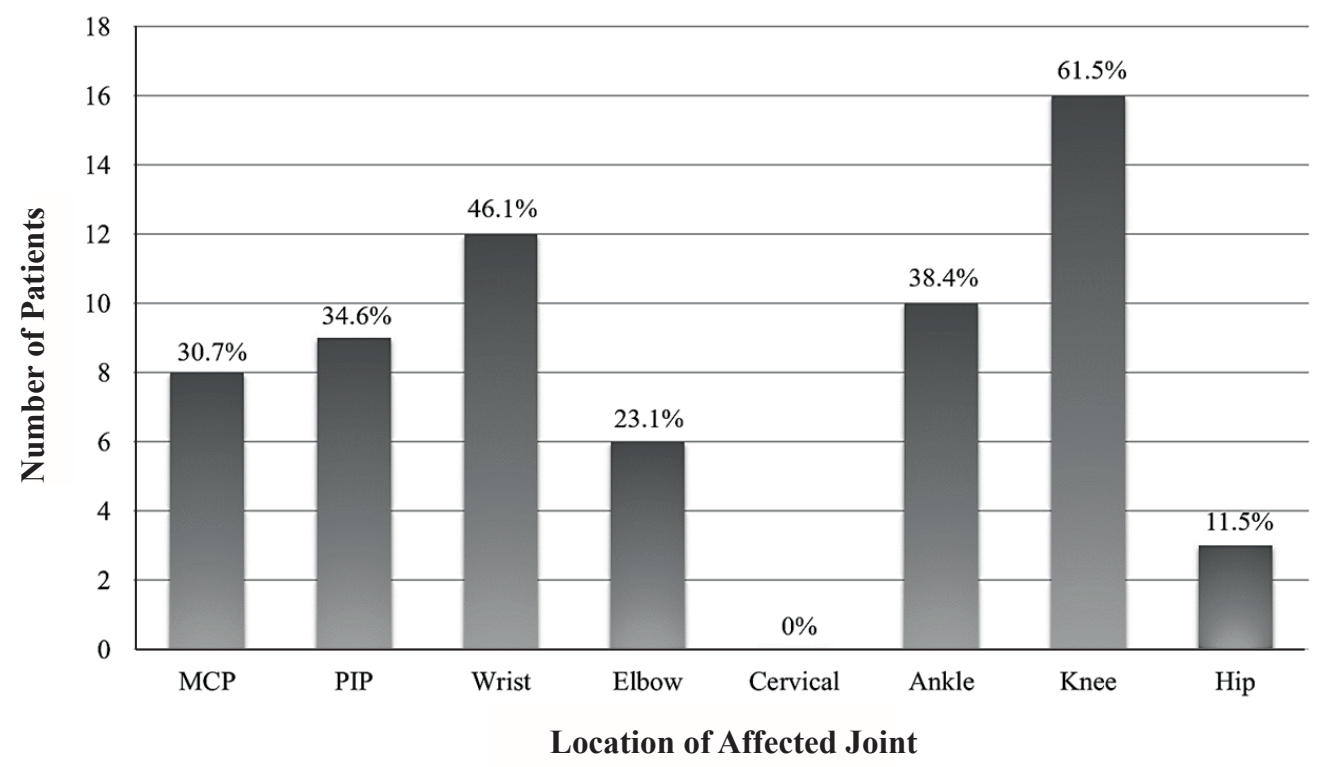

Figure 1. Location of affected joint in JIA patients. Data are expressed as frequency (\%). MCP: metacarpophalangeal joints, PIP: proximal interphalangeal.

rheumatoid factor-positive polyarthritis, 5 (19.2\%) subjects with rheumatoid factor-negative polyarthritis, and $1(3.8 \%)$ subject with systemic arthritis. The most common clinical manifestation in JIA patients was arthritis, followed by fever and skin rash. The number of involved joints varied from 1-22 joints. The most commonly affected joints were the knee, wrist, and ankle (Figure 1). Six (23.1\%) subjects were receiving only nonsteroidal anti-inflammatory drugs (NSAIDs) as treatment, 11 (42.3\%) subjects were using a combination of NSAIDs and corticosteroid, and 9 (34.6\%) subjects were taking disease-modifying anti-rheumatic drugs (DMARDs) such as methotrexate in addition to NSAIDs and corticosteroid.

The average JADAS-27 score was 14.14 \pm 4.24 (range 8.00-24.40). Based on the JADAS-27 scores, all JIA subjects in this study were considered to have active disease and high disease activity. The highest JADAS-27 scores were found in subjects with systemic arthritis. The components of JADAS-27 (patient's VAS, physician's VAS, joints with active disease, ESR) and disease activity classification are described in Table 2. JADAS-27 scores were significantly different among the three groups divided by vitamin $\mathrm{D}$ levels ( $p=0.001$ ). JADAS-27 scores were highest in the vitamin D deficiency group and lowest in the sufficiency group (Figure 2 ). There was a statistically significant negative correlation between vitamin D levels and JADAS-27 scores $(p=0.001$, $\mathrm{r}=-0.595)$.

We also examined the correlation between vitamin D serum levels with JADAS-27 components. There were statistically significant negative correlations between vitamin D serum levels and the patient's VAS and physician's VAS ( $p=0.001$ and $p=0.000$, respectively). No association was found between vitamin $\mathrm{D}$ serum levels and affected joint count or $\operatorname{ESR}$ ( $p=0.116$ and $p=0.127$, respectively).

Serum CRP levels were elevated in JIA patients compared to healthy control subjects $(3.34 \pm 3.65 \mathrm{mg} / \mathrm{L} v s$. $0.72 \pm 0.57 \mathrm{mg} / \mathrm{L}, p=0.000$ ) (Table 1). No difference was

Table 2. JADAS-27 measurement in JIA patients $(\mathrm{n}=\mathbf{2 6})$.

\begin{tabular}{lc}
\multicolumn{1}{c}{ Parameters } & Value \\
\hline JADAS-27 component & \\
Patient's VAS & $4.58 \pm 1.24$ \\
Physician's VAS & $4.31 \pm 1.05$ \\
Joint count & $3.88 \pm 2.32$ \\
ESR normalized & $1.37 \pm 1.18$ \\
Total JADAS-27 & $14.14 \pm 4.24$ \\
\hline JADAS-27 in each JIA subtypes & \\
Oligoarthritis (n=12) & $11.07 \pm 2.07$ \\
RF-positive polyarthritis ( $\mathrm{n}=8)$ & $17.95 \pm 4.33$ \\
RF-negative polyarthritis $(\mathrm{n}=5)$ & $14.54 \pm 2.32$ \\
Systemic arthritis ( $\mathrm{n}=1)$ & 18.6 \\
\hline Classification of JADAS-27, $\mathrm{n}$ & \\
Inactive disease & 0 \\
Low disease activity & 0 \\
Moderate disease activity & 0 \\
High disease activity & $26(100 \%)$ \\
\hline
\end{tabular}

Results are expressed as mean $\pm \mathrm{SD}$ and frequency (\%). JADAS-27: Juvenile Arthritis Disease Activity Score 27; VAS: Visual Analog Scale; ESR: Erythrocyte Sedimentation Rate; JIA: Juvenile Idiopathic Arthritis; RF: Rheumatoid factor. 


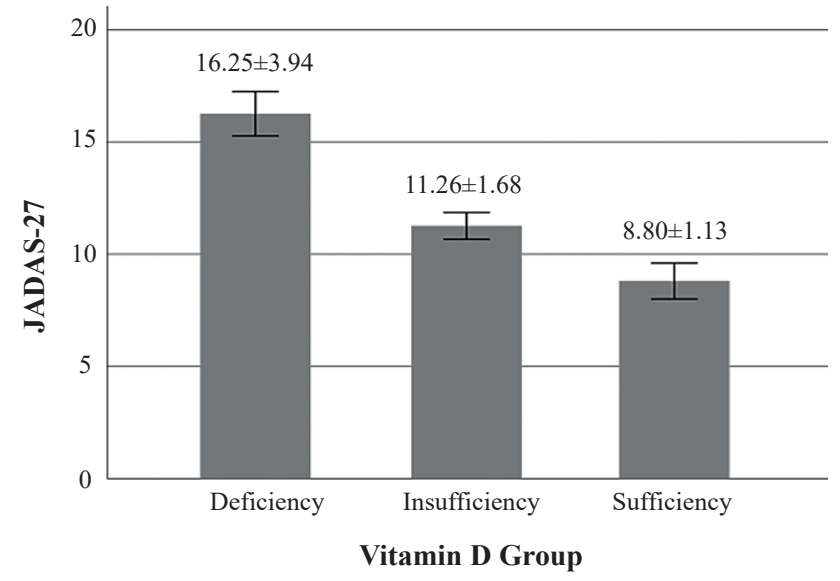

Figure 2. Comparison of JADAS-27 in different vitamin D classification. ANOVA and Post Hoc test were used to analyze the different of mean the JADAS-27 in each group.

found between the CRP serum levels among vitamin D group ( $p=0.441$ ). But the mean of CRP serum level was highest in the vitamin D deficiency group and lowest in the sufficiency group (Figure 3). There was also significant inverse correlation between vitamin $\mathrm{D}$ and CRP serum level $(p=0.021, \mathrm{r}=-0.452)$. CRP is an inflammatory marker that increased in chronic inflammatory condition such as in JIA. We found a significant positive association between CRP serum level and JADAS-27 ( $p=0.016, r=0.468)$. The correlation between vitamin D, CRP and disease activity in JIA patients can be found in Figure 4. In multivariate linear regression analysis, a similar association was found after adjusting for age, BMI and thrombocyte count $(p=0.014$ for vitamin $\mathrm{D}, p=0.027$ for CRP and $p>0.05$ for other parameters).

\section{Discussion}

In this study, there were 26 JIA patients enrolled in three years of observation. Among the patients, $57.6 \%$ were girls and $42.4 \%$ were boys, with the mean age was $12.92 \pm 1.62$ years old, which is not substantially different from other studies.(22) A previous study reported that 28 patients with JIA were seen at the Dr. Hasan Sadikin General Hospital in Bandung Indonesia between January 2006 and October 2011, which is consisted of 10 boys and 18 girls with ages ranging from 2 to 14 years.(23) We found that the majority of patients with JIA had the oligoarthritis subtype, followed by rheumatoid factor-positive polyarthritis and that the most commonly affected joints were the knee, wrist, and ankle. Similar studies in the USA, Germany, Brazil, Morocco, and Turkey also reported that oligoarticular JIA is the most common JIA subtype.(9-13) There was a high incidence of underweight nutritional status in patients with JIA in our study. There seems to be an independent association between being underweight and higher disease activity in JIA patients.(24) A cross-sectional study conducted in Norway showed that there was no difference in height or BMI between JIA patients and healthy subjects. However, a significantly reduced BMI was observed in female JIA patients with the systemic disease compared to those with oligoarticular JIA. In addition, female JIA patients who had been using corticosteroids had significantly lower weight, height, and BMI than patients who had never used corticosteroids.(25) Importantly, these results suggest that JIA could impair a child's nutritional status.

There was a low level of vitamin D in the group of JIA patients compared to the age- and sex-matched control group. Several other studies also reported that there were low levels of vitamin D in children with JIA compared to healthy control subjects. $(6,10,26)$ However, a meta-analysis including 19 studies reporting values of $25(\mathrm{OH}) \mathrm{D}$ and $1,25(\mathrm{OH}) \mathrm{D}$ in patients with JIA could not find evidence to link vitamin D deficiency with JIA due to the lack of cutoff criteria of vitamin D deficiency in pediatric populations. $(5,17)$ The American Academy of Pediatrics recommended that serum concentrations of $25(\mathrm{OH}) \mathrm{D}$ should be maintained above $20 \mathrm{ng} / \mathrm{mL}$ in children, but it did not establish the values to distinguish between sufficiency and insufficiency. (18) The Endocrine Society has published guidelines stating that the optimal 25(OH)D concentration in children is above $75 \mathrm{nmol} / \mathrm{L}$ (30 ng/mL).(19) The South East Asian Nutrition Surveys (SEANUTS), a cross-sectional nutritional survey including four South East Asian countries, reported that the mean level of 25(OH)D in Indonesian children aged 0.5 to 12 years was $52.7 \mathrm{nmol} / \mathrm{L}(21.08 \mathrm{ng} / \mathrm{mL})$. There was not

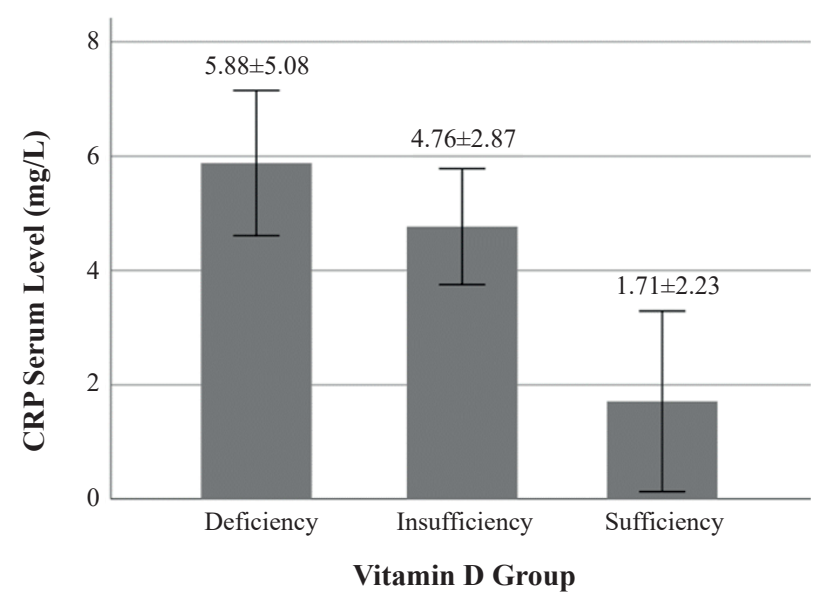

Figure 3. Comparison of CRP serum level in different vitamin D classification. ANOVA and Post Hoc test were used to analyze the different of mean the CRP in each group. 
A.

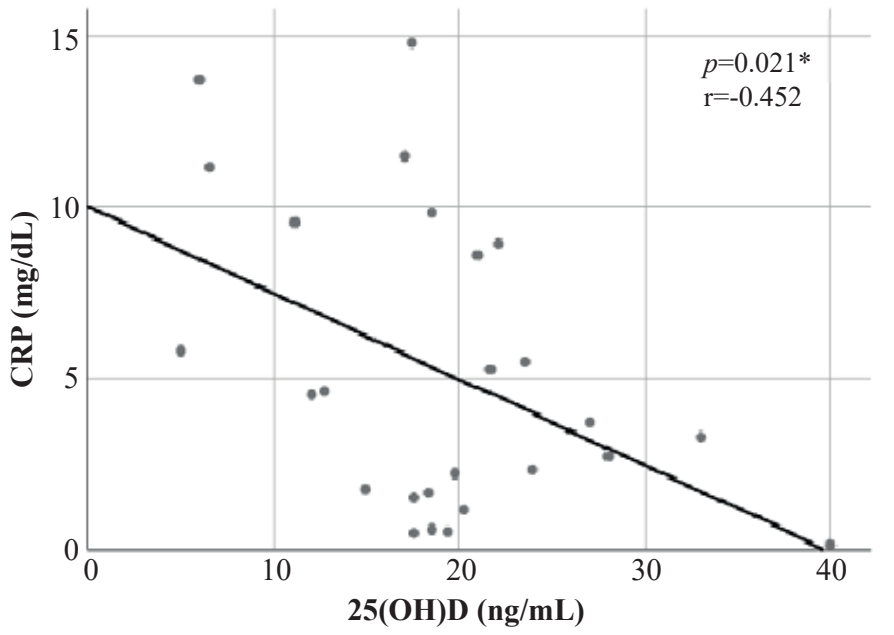

B.

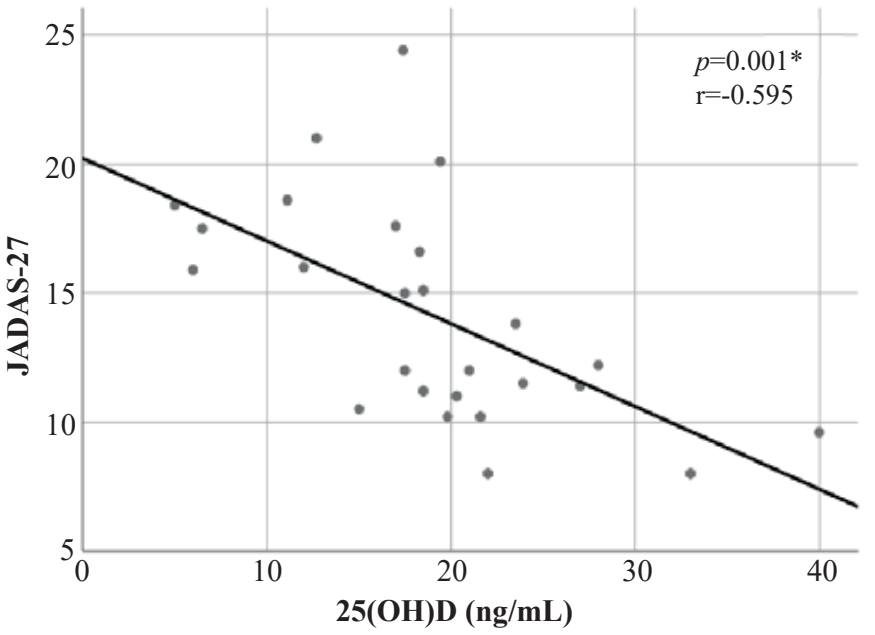

C.

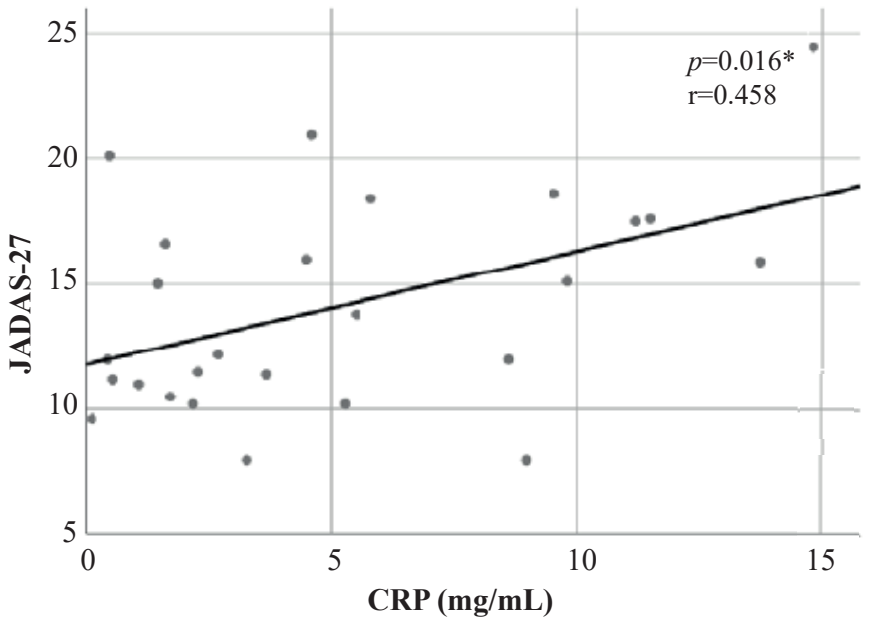

Figure 4. Correlation between Vitamin D, CRP and disease activity in JIA patients. A: correlation between CRP levels and JADAS-27 scores; B: correlation between vitamin D levels and JADAS-27 scores; C: correlation between CRP levels and JADAS-27 scores. yet a standard classification of sufficient, insufficient, and deficient vitamin D levels.(27) A previous study conducted in Jakarta, Indonesia, involving 120 children aged 7-12 years from two different elementary schools used three classifications of $25(\mathrm{OH}) \mathrm{D}$ levels: sufficient if $\geq 32 \mathrm{ng} / \mathrm{dL}$, insufficient if between 15 and $31 \mathrm{ng} / \mathrm{dL}$, and deficient if $<15$ ng/dL.(28) Prior to this study, no studies had reported vitamin D levels in JIA patients in Indonesia.
It has been established that vitamin $\mathrm{D}$ has an essential role in the pathogenesis of autoimmune and rheumatic diseases.(29) These effects can be achieved only if $(1,25(\mathrm{OH}) 2 \mathrm{D})$, the active form of vitamin $\mathrm{D}$ that is generated from circulating 25-hydroxyvitamin D $(25(\mathrm{OH})$ $\mathrm{D}$ ), is bound to the vitamin $\mathrm{D}$ receptor (VDR). VDR then binds to the vitamin $\mathrm{D}$ response element to activate the transcription of genes that regulate the inflammatory 
response. Vitamin D could inhibit the differentiation and maturation of human dendritic cells; suppress $\mathrm{T}$ helper 1 (Th1 and Th17 proliferation; promote Th2 and T regulatory cell proliferation; inhibit the synthesis, secretion, and release of pro-inflammatory cytokines; and induce the production of anti-inflammatory cytokines.(30) Consequently, low vitamin $\mathrm{D}$ concentrations are associated with higher disease severity in JIA.

A previous study showed that subjects with deficient levels of vitamin D produced higher levels of CRP and proinflammatory cytokines.(31) A meta-analysis reported that vitamin D supplementation had no effect on serum CRP and inflammatory cytokines.(16) In the present study, there was a high CRP serum level in JIA patients and a positive correlation between CRP levels and JADAS-27 scores; thus, high CRP levels were associated with high JADAS-27 scores. The American College of Rheumatology developed consensus guidelines for JIA treatment, including the use of CRP and ESR as biomarkers. A high CRP level, greater than twice the upper limit of normal, is considered a poor prognostic indicator in patients with JIA.(32) A crosssectional study conducted in Turkey found that a low level of vitamin D correlates with high disease activity in JIA patients.(10) Other studies also confirmed that JIA patients with active disease or frequent relapses had significantly lower levels of 25(OH)D compared to patients with no active disease or infrequent flares.(26) In contrast with our results, other studies reported no correlation between vitamin D level and JIA subtypes or disease activity.(11,13) A similar study reported that the vitamin D serum levels in children with JIA were significantly lower than those of healthy children, but there was no significant difference between vitamin D levels during active JIA and JIA in remission. They also found no significant correlation between disease activity and serum vitamin D levels.(6) A case-control study in China also reported no correlation between vitamin D serum level and JIA subtypes, CRP and ESR.(14) A study examining serum 25(OH)D levels and disease activity in children and adolescents in the US with JIA performed also found no association between vitamin $\mathrm{D}$ and disease activity.(15) These different results may be due to different methodological approaches, the different geographic regions studied, and the demographically disparate populations.(2) The limitations of the present study are relatively small sample size, single-center study and there was no data about dietary intake of vitamin $\mathrm{D}$, daily topical sunscreen utilization, daily physical activities, and degree of exposure to the sun, all of which may affect vitamin D levels and a dose of treatment. Therefore, further studies are needed to confirm our findings.

\section{Conclusion}

In conclusion, the frequencies of vitamin D deficiency and insufficiency were considerably high among patients with JIA. Vitamin D had inverse correlation between with CRP and disease activity in JIA patients. Therefore, clinicians should be aware of vitamin D deficiencies in JIA patients and to consider vitamin $\mathrm{D}$ supplementation as an adjunctive treatment in JIA.

\section{Acknowledgements}

This study was supported by Pediatric Department Saiful Anwar Hospital and LAURA (Lupus, Autoimmune and Rheumatology) research group, Faculty of Medicine, Brawijaya University, Indonesia.

\section{References}

1. Yang, CY, Leung PS, Adamopoulos IE, Gershwin ME. The implication of vitamin D and utoimmunity: a comprehensive review. Clin Rev Allergy Immunol. 2013; 45: 217-26.

2. Giancane G, Alessandro C, Stefano L, Sergio D, Benedetta S, Angelo R. Juvenile idiopathic arthritis: diagnosis and treatment. Rheumatol Ther. 2016; 3: 187-207.

3. Barut K, Adrovic A, Şahin S, Kasapçopur O. Juvenile idiopathic arthritis. Balkan Med J. 2017; 34: 90-101.

4. Harrold LR, Craig S, Stanford S, Jeffrey RC, Maryam MA, Joel MG, et al. Incidence and prevalence of juvenile idiopathic arthritis among children in a managed care population, 1996-2009. J Rheumatol. 2013; 40: 1218-225.

5. Nisar MK, Masood F, Cookson P, Sansome A, Östörhave AJK. What do we know about juvenile idiopathic arthritis and vitamin D? A systematic literature review and meta-analysis of current evidence. Clin Rheumatol. 2013; 32: 729-34.

6. Dagdeviran-Cakir A, Arvas A, Barut K, Gür E, Kasapçopur O. Serum vitamin $D$ levels during activation and remission periods of patients with juvenile idiopathic arthritis and familial Mediterranean fever. Turkish J Pediatr. 2016; 58: 125-31.

7. Alhomaidah D, Alsagheir A, Al-mayouf SM. Coexistence of endocrinopathies in children with rheumatic diseases. Int J Pediatr Adolesc Med. 2016; 3: 119-22.

8. Finch SL, Alan MR, Hassan V. Vitamin D and juvenile idiopathic arthritis. Pediatric Rheumatology. 2018; 16: 34. doi: 10.1186/ s12969-018-0250-0.

9. Sengler C, Zink J, Klotsche J, Niewerth M, Liedmann I, Horneff $\mathrm{G}$, et al. Vitamin D deficiency is associated with higher disease activity and the risk for uveitis in juvenile idiopathic arthritis - data from a German inception cohort. Arthritis Res Ther. 2018; 20: 27684.

10. Comak E, Doğan CS, Uslu-Gökçeoğlu A, Akbaş H, Özdem S, Koyun $\mathrm{M}$, et al. Association between vitamin D deficiency and disease activity in juvenile idiopathic arthritis. Turkish J Pediatr. 2014; 56: 626-31. 
11. Azizieh F, Alyahya KO, Raghupathy R. Association between levels of vitamin $\mathrm{D}$ and inflammatory markers in healthy women. J Inflamm Res. 2016; 9: 51-7.

12. Bouaddi I, Rostom S, Badri DE, Hassani A, Chkirate B, Abouqal R, et al. Vitamin D concentrations and disease activity in Moroccan children with juvenile idiopathic arthritis. BMC Musculoskeletal Disorders. 2014; 15: 1-18.

13. De Sousa-Studart SA, Leite AC, Marinho AL, Pinto AC, Rabelo Júnior CN, de Melo NR, et al. Vitamin D levels in juvenile idiopathic arthritis from an equatorial region. Rheumatol Int. 2015; 35: 1717-23.

14. Wang Y, Lu MP, Teng LP, Guo L, Xu YP, Zou LX, et al. Association of vitamin D concentrations with juvenile idiopathic arthritis. Zhongguo Dang Dai Er Ke Za Zhi. 2015; 17: 375-8.

15. Pelajo CF, Lopez-Benitez JM, Kent DM, Price LL, Mille LC, Dawson-Hughes B. 25-hydroxyvitamin D levels and juvenile idiopathic arthritis: is there an asscociation with disease activity? Rheumatol Int. 2012; 32: 3923-9.

16. Mazidi $M$, Rezaie $P$, Vatanparast $H$. Impact of vitamin $D$ supplementation on C-reactive protein; a systematic review and meta-analysis of randomized controlled trials. BMC Nutrition. 2018; 4: 1. doi: 10.1186/s40795-017-0207-6.

17. Institute of Medicine. Food and nutrition board, dietary reference intakes for calcium and vitamin D. Washington: National Academy Press; 2010.

18. Romero MJ, Tejada G, Henríquez S. Vitamin D deficiency in childhood. Rev Osteoporos Metab Miner. 2017; 9 (Supl 1): S16-23.

19. Holick MF, Binkley NC, Bischoff-Ferrari HA, Gordon CM, Hanley DA, Heaney RP, et al. Evaluation, treatment, and prevention of vitamin D deficiency: an Endocrine Society clinical practice guideline. J Clin Endocrinol Metab. 2011; 96: 1911-30.

20. Mourou AF, Santos MJ, Melo-Gomes J, Martins FM, Costa JA, Ramos $\mathrm{F}$, et al. Using the juvenile arthritis disease activity score based on erythrocyte sedimentation rate od C-reactive protein level: results from the register. Arthritis Care Res. 2014; 66: 585-91.

21. Consolaro A, Giancane G, Schiappapietr B, Davì S, Calandra S, Lanni $\mathrm{S}$, Ravelli A. Clinical outcome measures in juvenile idiopathic arthritis. Pediatr Rheumatol. 2016; 14: 23. doi: 10.1186/s12969016-0085-5.
22. Al-Hemairi MH, Albokhari SM, Muzaffer MA. The pattern of juvenile idiopathic arthritis in a single tertiary center in Saudi Arabia. Int J Inflam. 2016; 2016: 7802957. doi: 10.1155/2016/7802957.

23. Ghrahani R, Setiabudiawan B, Sapartini G, Puspasari H. Distribution of juvenile idiophatic arthritis subtypes in Bandung. MKB. 2012; 44: 101-5.

24. Neto A, Mourão AF, Oliveira-Ramos F, Gomes JM, Santos MJ, Campanilho-Marques R, et al. Body mass index and disease activity in Portuguese and Brazilian JIA patients: results from rheumatic disease Portuguese Register-Reuma. Ann Rheum Dis. 2019; 78: 1344.

25. Haugen MA, Lien G, Flatø B, Kvammen JA, Vinje O, Sørskaar D, Førre Ø. Minor impact of juvenile arthritis on nutritional status in young adult patients. Arthritis Care Res. 2002; 47: 623-9.

26. Stagi S, Bertini F, Cavalli L, Matucci-Cerinic M, Brandi ML, Falcini F. Determinants of vitamin D levels in children, adolescents, and young adults with juvenile idiopathic arthritis. J Rheumatol. 2014; 41: 1884-92.

27. Poh BK, Rojroongwasinkul N, Nguyen BKL, Sandjaja, Ruzita AB, Yamborisut $\mathrm{U}$, et al. 25-hydroxy-vitamin D demography and the risk of vitamin D insufficiency in the South East Asian Nutrition Surveys (SEANUTS). Asia Pac J Clin Nutr. 2016; 25: 538-48.

28. Soesanti F, Pulungan A, Tridjaja B, Batubara JRL. Vitamin D profile in healthy children aged 7-12 years old in Indonesia. Int J Pediatr Endocrinol. 2013; 2013(Suppl 1): P167. doi: 10.1186/1687-98562013-S1-P167.

29. Vojinovic J, Rolando C. Vitamin D-update for the pediatric rheumatologists. Pediatr Rheumatol. 2015; 13: 18. doi: 10.1186/ s12969-015-0017-9.

30. Dankers W, Colin EM, van Hamburg JP, Lubberts E. Vitamin D in autoimmunity: molecular mechanisms and therapeutic potential. Front Immunol. 2016; 7: 697. doi: 10.3389/fimmu.2016.00697.

31. Swart JF, de Roock S, Prakken BJ. Understanding inflammation in juvenile idiopathic arthritis: how immune biomarkers guide clinical strategies in the systemic onset subtype. Eur J Immunol. 2016; 46: 2068-77.

32. Ravelli A, Martini A. Early predictors of outcome in juvenile idiopathic arthritis. Clin Exp Rheumatol. 2003; 21 (Suppl. 31): S89-S93. 